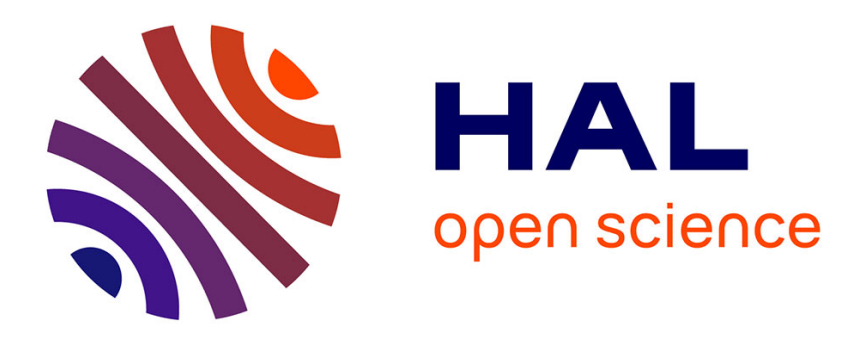

\title{
L'électrochimie des semi-conducteurs : le retour du silicium \\ J.-N. Chazalviel
}

\section{To cite this version:}

J.-N. Chazalviel. L'électrochimie des semi-conducteurs: le retour du silicium. Journal de Physique IV Proceedings, 1994, 04 (C1), pp.C1-117-C1-128. 10.1051/jp4:1994108 • jpa-00252450

\section{HAL Id: jpa-00252450 https://hal.science/jpa-00252450}

Submitted on 1 Jan 1994

HAL is a multi-disciplinary open access archive for the deposit and dissemination of scientific research documents, whether they are published or not. The documents may come from teaching and research institutions in France or abroad, or from public or private research centers.
L'archive ouverte pluridisciplinaire HAL, est destinée au dépôt et à la diffusion de documents scientifiques de niveau recherche, publiés ou non, émanant des établissements d'enseignement et de recherche français ou étrangers, des laboratoires publics ou privés. 


\title{
L'électrochimie des semi-conducteurs : le retour du silicium
}

\author{
J.-N. CHAZALVIEL
}

Laboratoire de Physique de la Matière Condensée, Ecole Polytechnique-CNRS, Route de Saclay, 91128 Palaiseau cedex, France

\begin{abstract}
Semiconductor electrochemistry has known an important development in the eighties, especially aimed to the photoelectrochemical conversion of solar energy. More fundamental work is now being addressed toward the most simple and well-known semiconductors, especially silicon. In spite of its propensity toward oxidation, silicon has demonstrated its suitability for realizing photoelectrochemical cells in non-aqueous solvents, with good stability and performance comparable to those of dry solid-state cells. Upon rinsing in fluoride medium, the silicon surface comes out covered with $\mathrm{Si}-\mathrm{H}$ bonds. In contrast to common expectation, this coating is stable on an hour time scale even in the presence of water. In a solvent such as methanol, the formation of $\mathrm{SiOCH}_{3}$ surface groups still reinforces surface stability. Chemical dissolution of silicon in alkaline media has long been known and used for its anisotropic properties. Recent studies have shown that, here again, the surface during the dissolution appears essentially hydrogenated, and the mechanism responsible for the anisotropy is being clarified. On the opposite, surface oxidation may be looked for, for example in view of the manufacturing of MOS devices or for the passivation of integrated circuits. Anodic oxidation may indeed provide oxides with a good electronic quality and improved thickness control. In fluoride media, significant work has been directed toward understanding the electrochemical dissolution processes of silicon. At moderate potentials, anodic dissolution may lead to the formation of porous silicon. This material, whose formation mechanism is still imperfectly understood, exhibits the characteristics of an original semiconductor. Especially, it may exhibit an intense luminescence in the visible range. This luminescence is presently arousing many excitement in view of its possible applications, but its origin is a matter of controversy. For more positive potentials, the anodic dissolution of silicon in fluoride media exhibits a polishing regime. For the most positive potentials, an original resonant electrical behavior of the interface has been observed. This behavior arises from an oscillating behavior on the microscopic scale. The origin of this oscillation, associated to a variation of the interfacial oxide thickness, is still to be elucidated.
\end{abstract}

\section{Introduction.}

La photoélectrochimie sur électrodes semi-conductrices a connu un développement très rapide dans les années 1980. Ces recherches se sont développées essentiellement dans le but de réaliser la conversion photoélectrochimique de l'énergie solaire. Afin de résoudre le problème difficile de la corrosion des électrodes, des centaines de matériaux ont été testés comme photoélectrodes. Certains d'entre eux ont d'ailleurs montré des propriétés prometteuses. Néanmoins, la difficulté d'égaler la stabilité des dispositifs solides et la baisse sensible d'intérêt pour le solaire ont amené un déclin de cette activité [1].

Toutefois, l'expérience acquise sur l'électrochimie des semi-conducteurs a produit diverses retombées. Parmi d'autres domaines, la photocatalyse a bénéficié de ces connaissances [2]. En sciences des matériaux, on a exploité la facilité de réaliser des jonctions liquides pour caractériser des semi-conducteurs nouveaux [3], des oxydes anodiques [4], ou des semi-conducteurs pour la technologie [5]. Enfin, nombre de groupes ont été amenés à s'intéresser à la chimie de surface des semi-conducteurs en milieu humide. Ce domaine est évidemment d'un intérêt capital pour la technologie des dispositifs, où les étapes humides sont inévitables, et cependant les connaissances y apparaissent étonnamment réduites. Ce tournant a amené les travaux à se 
focaliser davantage sur un petit nombre de semi-conducteurs présentant un intérêt technologique pour l'électronique. Ainsi, une activité importante se poursuit actuellement sur les composés III-V [6]. Néanmoins, l'aspect sans doute le plus spectaculaire de ce tournant est le nombre considérable d'études se déroulant à l'heure actuelle sur ce matériau en principe si bien connu qu'est le silicium.

Nous présenterons d'abord les résultats essentiels obtenus sur le silicium en matière de photoélectrochimie (Section 2). Ces résultats, obtenus en milieu non aqueux, nous amèneront à faire le point sur l'état actuel de nos connaissances sur la chimie de surface du silicium, en milieu aqueux, fluorure ou alcalin, et en milieu organique (Sections 3-5). Dans une deuxième partie, nous nous intéresserons à la réaction d'oxydation anodique sur silicium en milieu aqueux. En l'absence d'ions fluorure, cette oxydation conduit à la formation d'une couche passive (Section 6). En milieu fluorure, différents régimes sont possibles. Aux faibles potentiels, on obtient du silicium poreux, matériau qui suscite actuellement un intérêt considérable en raison de ses propriétés extraordinaires de luminescence (Section 7). Aux potentiels plus positifs, on observe un régime de polissage, dont les caractéristiques originales présentent un grand intérêt fondamental (Section 8).

\section{Piles photoélectrochimiques basées sur le silicium.}

La réaction d'oxydation anodique du silicium en milieu aqueux est caractérisée par un potentiel assez négatif $(\sim-0.8 \mathrm{~V}$ par rapport à NHE à $\mathrm{pH}=0$ [7]). Cette tendance à s'oxyder facilement en présence d'eau a longtemps découragé les photoélectrochimistes de s'intéresser à ce matériau. L'intuition commune indique que l'oxydation chimique tendra à former à la surface du silicium une couche de silice, qui est connue pour être un oxyde particulièrement bloquant. La situation est bien pire lorsqu'on prétend faire fonctionner une photoanode en silicium de type $n$, car à la réactivité chimique s'ajoute alors la possibilité d'un processus d'oxydation anodique. Les premières tentatives en milieu non aqueux se sont d'ailleurs elles-mêmes avérées décevantes: par exemple, dans l'acétonitrile, on pourrait espérer ralentir l'oxydation de l'électrode en éliminant au mieux l'eau résiduelle, et la protéger encore davantage en incorporant à l'électrolyte une espèce oxydable et à cinétique rapide telle que le ferrocène. Cette double stratégie a été utilisée avec succès sur nombre de photoanodes semi-conductrices [8]. Dans le cas du silicium, même pour des teneurs en eau aussi faibles que quelques ppm, une telle photoanode ne fonctionne guère au-delà de quelques minutes sous un éclairement typique [9]: l'eau tend à se physisorber préférentiellement à la surface de l'électrode [10]; lorsqu'un trou atteint cette surface, la réaction d'oxydation s'effectue facilement et l'oxyde formé passive rapidement la surface.

Toutefois, une percée décisive a été effectuée en 1984, lorsque l'équipe de Lewis choisit d'utiliser le méthanol comme solvant. Dans des électrolytes du type [méthanol $+\mathrm{LiClO}_{4}+$ diméthylferrocène / diméthylferricinium], une photoélectrode de silicium $n$ peut permettre de réaliser une photopile présentant un rendement de 14\%, comparable aux rendements obtenus pour les photopiles solides (jonctions pin), et stable à l'échelle de plusieurs mois $[11,12]$. Bien qu'une telle durée soit encore loin des performances nécessaires à la faisabilité d'un dispositif industriel, cette étonnante amélioration de stabilité suggère l'existence d'un effet spécifique à l'interface silicium/méthanol, sur lequel nous reviendrons ultérieurement. De plus, ces photopiles présentent un rendement quantique bien constant jusque dans le bleu, ce qui indique que la recombinaison de surface est étonnament faible. Enfin, l'utilisation de silicium polycristallin ne fait chuter le rendement qu'à $10 \%$, performance remarquable au regard des dispositifs solides.

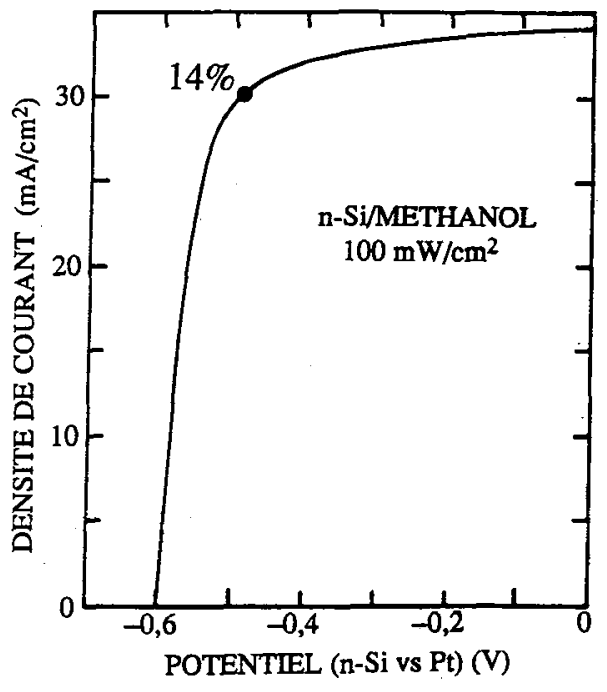

Fig.1.- Performance typique d'une cellule $n$-Si/methanol (điméthylferrocène/diméthylferricinium)/Pt à couche mince d'électrolyte $(\sim 10 \mu)$ (d'après [11]). 


\section{Surface du silicium rincé en milieu fluorure.}

L'acide fluorhydrique, un des rares milieux où la silice est soluble, est utilisé depuis longtemps pour préparer des surfaces de silicium. Un échantillon de silicium rincé dans HF en sort avec une surface hydrofuge. Au début des années 1970, Beckmann avait montré, par spectroscopie infrarouge, que cette surface apparaît couverte de liaisons $\mathrm{Si}-\mathrm{H}$, ce qu'il interprétait comme une couche de type polymère ("polymerized silene") [13]. Ce résultat est passé relativement inaperçu et ce n'est que dans les années 1980 que d'autres études l'ont confirmé et l'ont peu à peu fait accepter par la communauté [14-17]. En fait, les études très détaillées menées par les équipes de Burrows et Chabal montrent que la surface du silicium rincé dans HF concentré est entièrement saturée par une monocouche d'hydrogène, mais est rugueuse à l'échelle atomique, comme l'atteste la présence de groupements $\mathrm{SiH}, \mathrm{SiH}_{2}, \mathrm{SiH}_{3}$ [17]. Ces groupements sont clairement identifiés par leurs spectres d'absorption infrarouge (région $2080-2150 \mathrm{~cm}^{-1}$ ), facilement distinguables et eux-mêmes séparés en différentes sous-contributions, correspondant à des environnements différents de l'atome de silicium du groupement. En revanche, pour des pH élevés (par exemple solution $\mathrm{NH}_{4} \mathrm{~F}$ tamponnée vers $\mathrm{pH} 10$ ), le rinçage conduit à une surface de silicium non reconstruite, atomiquement plane et hydrogénée, caractérisée par un pic vibrationnel vSiH exceptionnellement étroit (largeur à mihauteur inférieure à $1 \mathrm{~cm}^{-1}$ à température ambiante) [18]. II s'agit là de la surface de silicium la plus proche de l'idéalité qu'on ait pu préparer. Cette surface a été utilisée comme système modèle par les physiciens des surfaces et a donné lieu à de nombreuses études (LEED, STM, EELS, études vibrationnelles fines: génération de fréquence somme, études résolues en temps menant à la détermination des temps de déphasage et de relaxation vibrationnelle, etc...)[19-22]. Il est remarquable que la vitesse de dissolution chimique du silicium en milieu fluorure, très faible dans l'acide fluorhydrique concentré, croît avec le pH du milieu. Ceci suggère un mécanisme catalysé par les ions $\mathrm{OH}^{-}$, et qui pourrait être anisotrope. L'obtention de surfaces atomiquement planes en milieu alcalin résulterait de l'anisotropie de ce mécanisme.

Cette surface hydrogénée s'avère étonnamment stable en présence d'eau. Ainsi, en électrolyte organique (acétonitrile + sel support +10 ppm $\mathrm{H}_{2} \mathrm{O}$ ), une étude infrarouge in-situ a montré que dès le début l'eau contenue dans l'électrolyte tend à se physisorber à l'interface (pic de déformation en ciseaux de la molécule d'eau $\delta \mathrm{OH}_{2}$ à 1650 $\mathrm{cm}^{-1}$ ), mais, dans l'obscurité et en l'absence de potentiel appliqué, les groupements $\mathrm{SiH}$ subsistent néanmoins à l'échelle de quelques dizaines d'heures [10]. Progressivement, on voit apparaître des groupements $\mathrm{SiOH}$ et SiOSi, ainsi qu'un pic $\mathrm{SiH}$ décalé en fréquence ( 2270 $\mathrm{cm}^{-1}$ ), correspondant à des atomes de silicium oxydés à l'arrière $\left(\mathrm{O}_{3} \mathrm{SiH}\right)$. Cette étude montre de plus que l'oxydation spontanée d'une surface [111] dans un tel milieu procède par formation d'îlots d'oxyde, de dimensions latérales typiques 20 A [10].

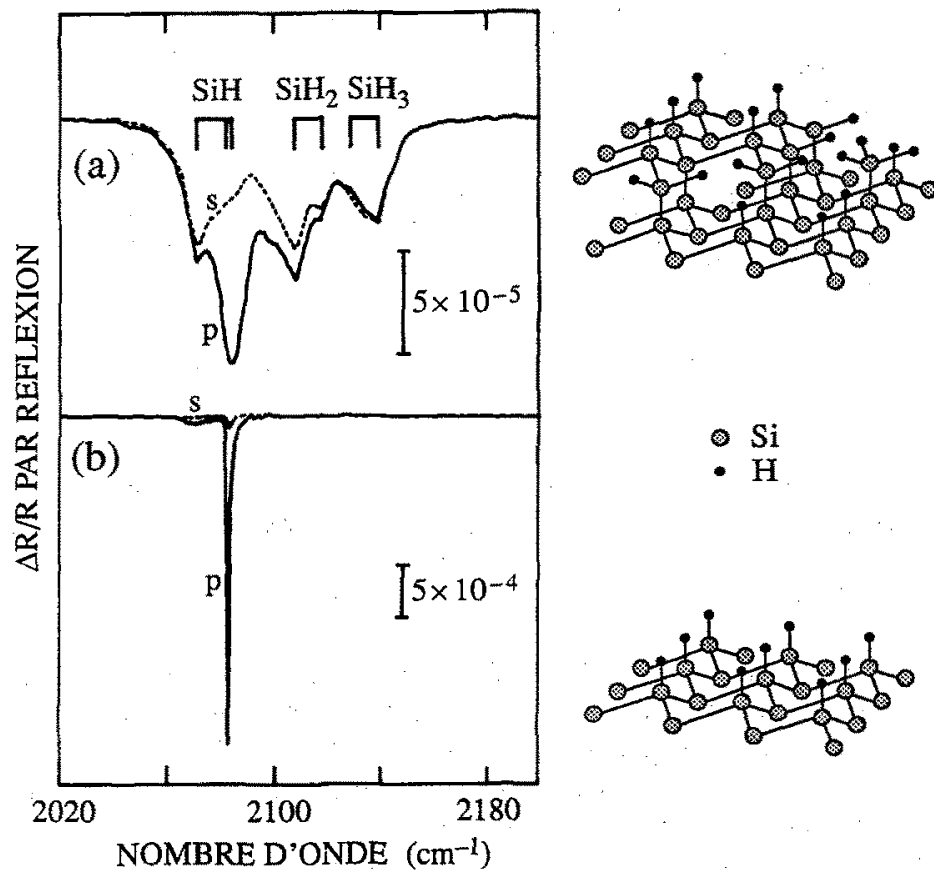

Fig.2.- Spectres infrarouge et représentation schématique d'une surface de silicium [111] (a) rincée dans HF, et (b) dans $\mathrm{NH}_{4} \mathrm{~F}$ à pH 10 (d'après [17] et [18]). Spectres de transmission diffêrentielle enregistrés en géometrie de multiréflexions internes (ATR), en polarisation p (courbes en trait plein) et $s$ (pointilles). 


\section{Modifications de surface en milieu organique.}

Les groupements $\mathrm{SiOH}$ à la surface de la silice sont bien connus pour pouvoir donner lieu à des greffages de groupements organiques [23]. On a montré que, de même que pour la silice, l'exposition de la surface du silicium à des vapeurs de chlorotriméthylsilane ou d'hexaméthyldisilazane conduit au greffage de groupements - $\mathrm{OSi}\left(\mathrm{CH}_{3}\right)_{3}$ [24,25]. Cette possibilité a été exploitée, soit pour tenter de stabiliser la surface vis-à-vis de l'oxydation [24,25], soit pour greffer des groupements électroactifs [26]. Ces greffages ont été effectués à l'aide d'un composé organosilicié, conduisant à un accrochage par l'intermédiaire d'un pont SiOSi.

Les performances exceptionnelles du silicium en tant que photoélectrode en milieu $\mathrm{CH}_{3} \mathrm{OH}$ ont également conduit à s'interroger sur la nature de sa surface dans ce milieu. Une étude infrarouge a montré qu'une surface de silicium exposée à du méthanol (liquide ou vapeur) se couvre partiellement de groupements $\mathrm{SiOCH}_{3}$ [25]. Une surface ainsi modifiée s'avère encore plus stable que la surface hydrogenée: l'interface formée par une surface ainsi prétraitée avec un électrolyte à base d'acétonitrile conserve des caractéristiques stables (en particulier faible densité d'états de surface) à l'échelle de plusieurs jours. De plus, une telle surface peut supporter des rinçages dans l'eau, en milieu acide ou basique, sans être appréciablement affectée. Enfin, la surface modifiée présente un décalage négatif du potentiel de bandes plates, qui permet d'expliquer les bons photopotentiels obtenus sur photoanodes de type $\mathrm{n}$ dans le méthanol. Toutefois, le rôle d'une lente dissolution chimique du silicium dans le méthanol (avec formation de tétraméthoxysilane) n'est sans doute pas complètement à exclure dans ce dernier cas.
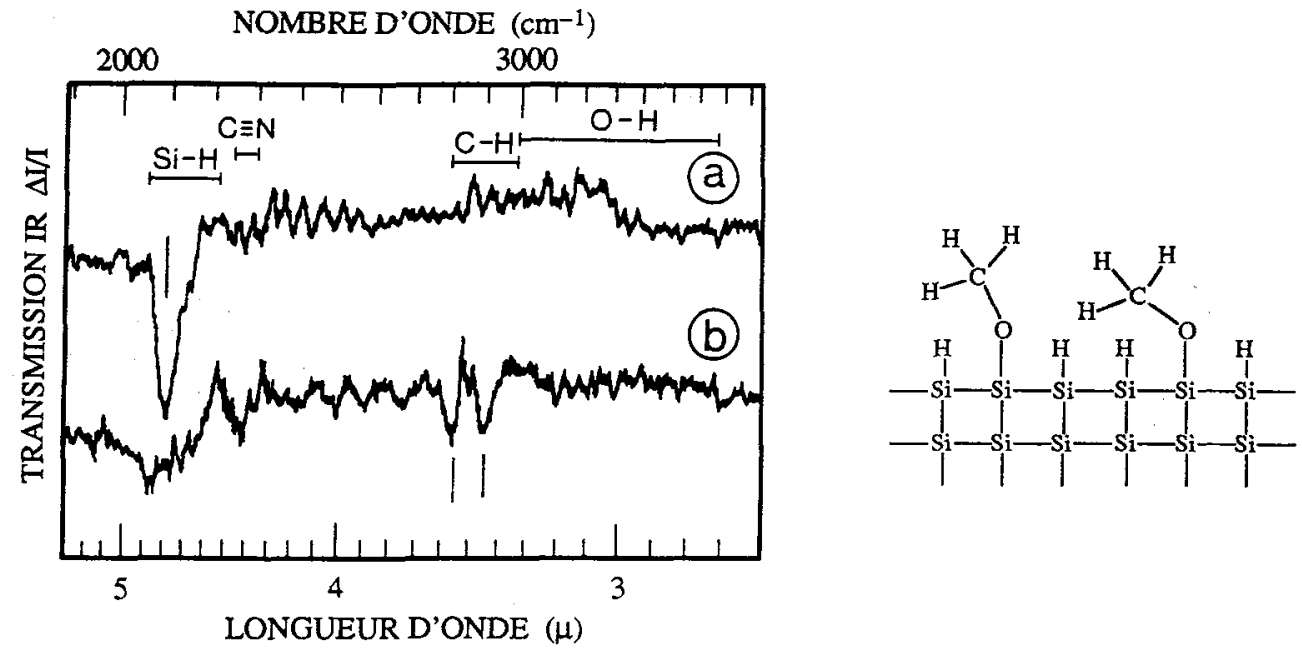

Fig.3.- Spectre infrarouge (obtenu in-situ dans l'acétonitrile en modulation de potentiel) d'une surface de silicium [111] (a) rincée dans $H F$ et (b) prétraitée au méthanol. A droite, représentation schématique de la surface ainsi modifiée (d'après [25]).

\section{Surface du silicium en milieu alcalin.}

La dissolution chimique du silicium en milieu alcalin est connue depuis longtemps pour ses propriétés remarquables d'anisotropie: la vitesse de dissolution des faces [111] est typiquement deux ordres de grandeur plus faible que pour les autres orientations [27,28]. Cette propriété est utilisée couramment par la technologie pour réaliser des micro-usinages. Les solutions utilisées sont généralement des mélanges éthylènediamine/pyrocatéchol/eau. Toutefois, les mêmes effets anisotropes sont également observés dans de simples solutions aqueuses de soude ou de potasse. L'effet d'anisotropie est evidemment à rapprocher de celui observé dans les solutions basiques de fluorures.

De fait, des études récentes ont montré que le mécanisme de dissolution apparaît très semblable que le milieu comporte ou non des ions fluorure [29,30]. Dans les deux cas, la surface des terrasses [111] 
apparaît non reconstruite et couverte d'hydrogène (bien que la signature infrarouge des liaisons SiH n'ait jusqu'ici été obtenue qu'ex-situ pour le milieu non fluorure). Ceci s'interprète naturellement dans le cadre d'un mécanisme où l'étape limitante est la rupture d'une liaison SiH superficielle (catalysée par les ions $\mathrm{OH}^{\sim}$ ); le groupement SiOH résultant polarise les liaisons Si-Si arrière, et celles-ci peuvent à leur tour être rompues par réaction rapide avec l'eau. Une fois l'atome de silicium oxydé parti en solution, la surface hydrogénée se trouve régénérée. Des études par microscopie tunnel in-situ ont montré que la dissolution est ralentie sous polarisation cathodique. L'anisotropie se trouve alors renforcée, la dissolution d'une face [111] s'effectuant uniquement par les marches. Aux potentiels intermédiaires, l'attaque peut également s'effectuer par formation de piqûres sur les terrasses [111]. Ces observations ont permis de proposer un mécanisme détaillé de la dissolution chimique et électrochimique du silicium en milieu alcalin [29,30].

(a)

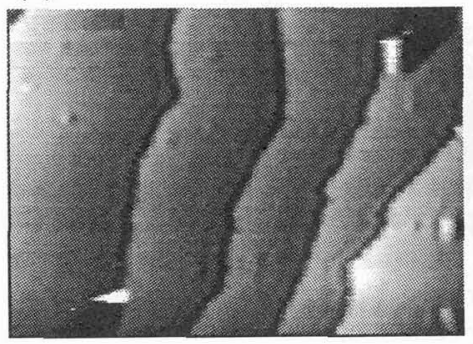

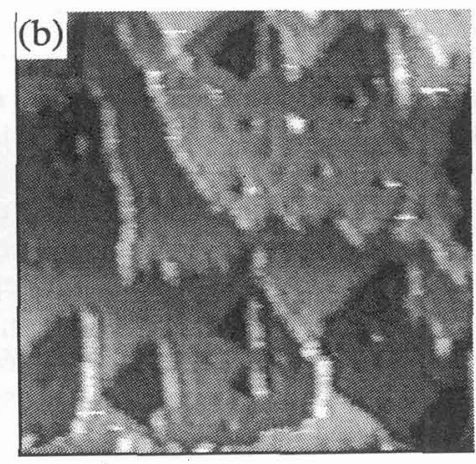

Fig.4.- Imagerie par STM in-situ d'une surface de silicium de type $n$ dans $2 \mathrm{M} \mathrm{NaOH}$, sous polarisation cathodique. (a) $J=-150 \mu \mathrm{A} / \mathrm{cm}^{2}$, (b) de même après prépolarisation à $J=0$ (noter la piqûration importante), (c) même surface que (a) montrée sur une aire réduite, permettant d'observer la surface $S i$ [111] $1 \times 1$ avec la résolution atomique. Dimensions des régions teprésentées: (a): $1270 \AA \times 935 \AA$, (b): $1100 \AA \times 1100 \AA$, (c): $50 \AA \times 50 \AA$ (d'après les auteurs de [29-30]).

\section{Oxydes anodiques sur silicium.}

L'oxydation du silicium, loin d'être une gêne comme en photoélectrochimie, est pour la technologie un phénomène essentiel. Les oxydes sont utilisés soit comme isolant dans les dispositifs à effet de champ (MOS), soit comme couche protectrice (passivation) sur les circuits intégrés. La silice anodique, bien qu'étudiée de longue date [31], a été délibérément ignorée par la technologie, pour des raisons de mise en œuvre, mais également parce que les premiers essais réalisés avaient fourni des oxydes très imparfaits, présentant en particulier des défauts d'interface. De tels défauts, dus aux ions de l'électrolyte [32], affectent gravement la qualité électronique des interfaces (états électroniques dans la bande interdite) et rendent de tels oxydes tout à fait inutilisables pour des structures MOS. L'oxydation thermique est donc devenue l'outil exclusif pour la réalisation des structures MOS et le dépôt par pulvérisation cathodique est le plus utilisé pour les oxydes de passivation.

Toutefois, des études plus récentes ont montré qu'il est possible de réaliser de "bons" oxydes anodiques, en utilisant en guise d'électrolyte de l'eau ultra-pure, en l'absence d'ions volontairement ajoutés [33]. Les oxydes obtenus ont des qualités comparables à celles des oxydes thermiques. De plus, le courant đ'oxydation dépend de façon critique de l'épaisseur d'oxyde déjà formé. Ceci permet une maitrise accrue de l'épaisseur de la couche et une homogénéité exceptionnelle. Cette technique pourrait être avantageuse pour la réalisation de couches très minces (dans la gamme inférieure à $100 \AA$ ) [33].

\section{Electrochimie du silicium en milieu fluorure: le silicium poreux.}

Les fluorures constituant l'un des rares milieux où la silice est soluble, les travaux dans ces milieux ont naturellement été nombreux, en particulier pour l'étude de la dissolution anodique du silicium. Comme c'est en général le cas pour les métaux, la dissolution anodique du silicium présente, à fort potentiel, un plateau 
de polissage (systématiquement pour le silicium de type $p$ ou $n^{+}$, seulement sous fort éclairement pour le type $n$ ), précédé d'une région à faible potentiel, où le courant augmente rapidement avec le potentiel $[34,35]$.

Comportement électrochimique a faible potentiel.-- Dans la région à faible potentiel, la dissolution d'un atome de silicium consomme 2 charges élémentaires (dissolution divalente) et la réaction électrochimique s'accompagne d'un dégagement d'hydrogène. Ceci suggère un mécanisme réactionnel en deux étapes: i) oxydation électrochimique avec deux charges, menant à un intermédiaire $\mathrm{Si}$, puis ii) étape chimique de réaction avec l'électrolyte $\left(\mathrm{Si}^{\mathrm{I}}+2 \mathrm{H}^{+} \rightarrow \mathrm{Si}^{\mathrm{NV}}+\mathrm{H}_{2}\right)$ avec éventuellement un chemin parallèle de dismutation $\left(2 \mathrm{Si}^{\mathrm{II}} \rightarrow \mathrm{Si}^{\mathrm{IV}}+\mathrm{Si}\right)$ [34]. Sur du type $n$, la réaction anodique ne se produit que sous éclairement. On observe alors un phénomène de doublement de courant, voire de quadruplement à faible éclairement [3638 (dans ce dernier cas la dissolution est tétravalente). Ceci indique qu'un seul trou est indispensable pour amorcer la réaction, les charges supplémentaires pouvant consister en électrons injectés dans la bande de conduction. Ce comportement est à rapprocher du courant anodique transitoire obtenu lorsqu'une couche d'oxyde parvient en fin de dissolution [36,39]. Il prouve la présence d'espèces intermédiaires fortement réductrices dans le mécanisme réactionnel. Un mécanisme analogue d'injection électronique est observé en présence de couples rédox oxydants [40].

Parmi les modèles réactionnels imaginés, beaucoup sont toutefois caducs, car la plupart postulaient un recouvrement de la surface par des liaisons $\mathrm{SiF}[34,41]$. Or, on a pu montrer que la surface dans ce régime reste essentiellement hydrogénée [42], tout comme au potentiel de repos. Le doublement de courant sur type $n$ a d'ailleurs pu être observé en transitoire en milieu non fluorure, sur du silicium préalablement rincé dans HF, suggérant que l'espèce intermédiaire réductrice est un intermédiaire d'oxydation des liaisons Si-H superficielles [43]. Les recherches d'intermédiaires réactionnels par spectroscopie in-situ n'ont jusqu'ici guère abouti et les mécanismes encore en lice restent dans l'attente de preuves expérimentales directes [44,45]. L'absence d'oxyde apparaît en tous cas naturelle au regard de l'expérience sur les métaux, où le régime de départ du courant anodique est en général attribué à une attaque directe de l'électrode (sans formation d'oxyde) et présente de plus un caractère rugosifiant pour la surface. Comme on va le voir, ce caractère rugosifiant est bien présent ici et se trouve même exalté de façon spectaculaire.

Le silicium poreux: fabrication et propriétés.- Dans des études anciennes, Uhlir [46] et Turner [47] avaient constaté que la dissolution anodique à faible potentiel pouvait conduire à la formation d'une couche superficielle colorée, pouvant atteindre une épaisseur de plusieurs microns, essentiellement constituée de silicium. L'étude microscopique de ces couches a montré qu'elles sont poreuses. Le comportement rugosifiant, classique sur les métaux, revêt ici un caractère extrême: l'électrode reste inchangée quant à son aspect géométrique (par exemple, épaisseur mesurée au comparateur), mais son aspect visuel est modifié (coloration). Ce changement est dû à la formation d'un fin réseau de canaux, sur une épaisseur proportionnelle à la charge faradique transférée, et qui peut atteindre plusieurs centaines de microns. Des études structurales montrent que la cristallinité du matériau apparaît en gros préservée. On a de fait transforme, par dissolution sélective de certaines régions, l'électrode de silicium massif en "silicium poreux" $[48,49]$.

Ce matériau peut être caractérisé par sa porosité (typiquement de $30 \%$ à $85 \%$ ) et la taille caractéristique des pores. Suivant la nature du cristal de départ $\left(n, p, n^{+}, p^{+}\right)$, la solution utilisée (généralement solution aqueuse de HF concentre, typiquement $15 \%$ à $50 \%$ ), et la densité de courant, on peut avoir une taille caractéristique de pores variant de $\sim 10 \AA \AA$ a $\sim 1 \mu$, mais plusieurs tailles seront généralement présentes au sein du même échantillon. Comme on peut s'y attendre, la porosité $P$ augmente lorsqu'on s'approche du plateau de polissage. Autrement dit, la porosité augmente lorsqu'on augmente le courant ou lorsqu'on diminue la concentration en HF. Toutefois, la variation de $P$ avec ces paramètres n'est pas simple et dépend du dopage du substrat. Un aspect remarquable est la très grande surface spécifique du matériau, de l'ordre de 500 à $1000 \mathrm{~m}^{2} / \mathrm{cm}^{3}$ sur substrat $p$. Cette grande valeur est clairement associée à la très faible taille des canaux les plus petits et explique la facilité d'oxydation thermique de ce matériau à des températures modérées. Cet aspect, et aussi la grande sélectivité du mécanisme de formation du poreux vis-à-vis du dopage du cristal de départ, ont attiré l'attention des technologues en vue de la réalisation de dispositifs pour l'électronique rapide (technologie SOI = "silicon on insulator" [48]). De plus, récemment, ce matériau s'est montré susceptible de présenter une photoluminescence intense dans le visible. L'intérêt potentiel de 
pouvoir réaliser des dispositifs optoélectroniques tout-silicium, ainsi que les questions fondamentales soulevées par cet effet inattendu, suscitent actuellement une activité intense sur ce sujet.

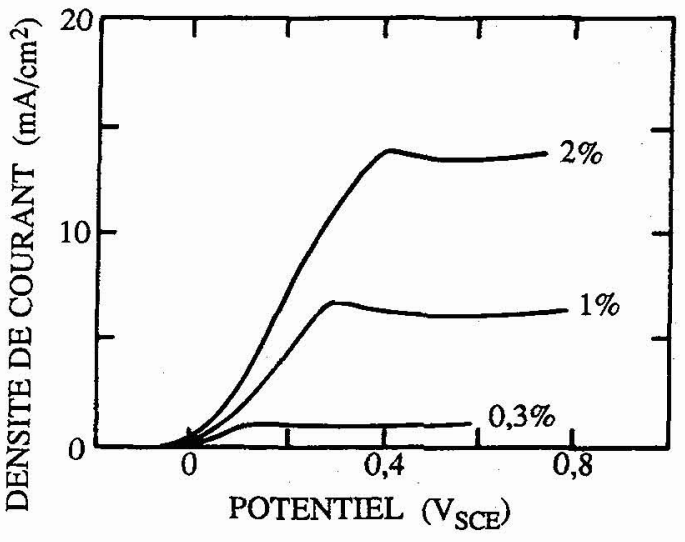

Fig.5.- Voltammogrammes typiques de $p$-Si dans HF de différentes concentrations $(1 \% \approx 0,5 \mathrm{M})$. La partie ascendante correspond au régime de génération du silicium poreux (d'après [35]).

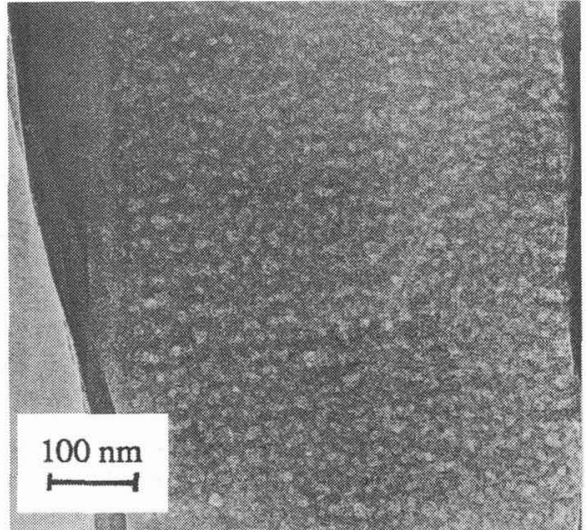

Fig.6.- Aspect d'une couche de silicium poreux sur Si de type $p$ peu dopé. Microscopie électronique à transmission (d'après les auteurs de [49b]).

Le mécanisme de formation du silicium poreux reste imparfaitement compris. Certains ont cherché à relier le mécanisme de formation du poreux au détail de la chimie du processus de dissolution [50]. Toutefois, il semble aujourd'hui que l'explication soit plutôt à rechercher du côté de la physique: le silicium poreux, une fois formé, est un matériau extrêmement résistif. On peut penser que ceci est dû soit à une dissolution sélective des dopants, soit plus vraisemblablement à un appauvrissement dû à la petite dimension des cristallites devant la longueur de Debye du matériau de départ. En tout état de cause, la résistivité du silicium poreux étant beaucoup plus grande que celle de l'électrolyte, on conçoit aisément que le courant faradique passera essentiellement dans l'électrolyte et la réaction se poursuivra au fond des pores. Cette idée permet bien d'expliquer qualitativement les comportements observés $[49,51]$, mais peut être compliquée par des effets quantiques dus aux petites tailles présentes dans le poreux [44], et une modélisation quantitative satisfaisante reste encore à élaborer.

Luminescence du silicium poreux.-Le silicium poreux a connu un regain d'intérêt considérable ces dernières années, lorsqu'il est apparu susceptible de donner lieu à une luminescence dans le visible.

La bande interdite du silicium $(1,1 \mathrm{eV})$ pourrait à première vue en faire un matériau luminescent dans l'infrarouge. Toutefois, pour des raisons de structure de bandes (gap indirect), la recombinaison radiative est très peu probable; en pratique, dans le silicium massif, les paires électron-trou se recombinent essentiellement de façon non radiative (centres de recombinaison), et seule une très faible luminescence peut être observée. C'est donc avec une grande surprise qu'on a constaté que du silicium poreux, formé sur substrat $p$, peut donner, à 1 'issue d'un traitement particulier (dissolution chimique partielle dans HF), une photoluminescence intense dans le visible [52]. D'autres traitements ont été mis au point depuis lors pour rendre luminescent le silicium poreux: oxydation anodique [53], oxydation lente à l'ambiante, ou oxydation thermique rapide à haute température (RTO) [54]. Les recherches se poursuivent à l'heure

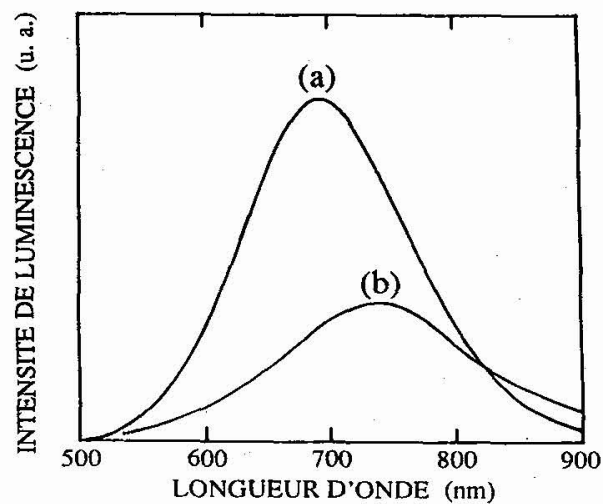

Fig.7.- Spectres typiques de photoluminescence de silicium poreux après oxydation anodique. Epaisseur $4 \mu$. Porosité initiale $85 \%$ (a) et $65 \%$ (b). 
actuelle dans le double but de comprendre l'origine de cette luminescence et de réaliser des dispositifs électroluminescents, seuls susceptibles d'applications.

Afin d'expliquer la photoluminescence du poreux après traitement chimique dans HF, Canham [52] a suggéré que la structure électronique du silicium est modifiée par la petite taille des cristallites de silicium constituant le poreux (particules ou plutôt filaments). Par des effets de confinement quantique, l'énergie des états de la bande de conduction serait augmentée, celle des états de la bande de valence abaissée, et l'augmentation obtenue du gap expliquerait le décalage vers le visible de la luminescence. Cette interprétation était soutenue à ses yeux par le décalage vers le bleu du spectre de luminescence lorsque la durée de dissolution dans HF est augmentée (plus les cristallites sont petits, plus les effets de confinement quantique sont importants). On conçoit de même que la petite taille des cristallites puisse relaxer quelque peu la règle de conservation du vecteur d'onde, qui interdit les transitions radiatives indirectes dans le silicium massif, et ainsi favoriser la luminescence par rapport aux processus non radiatifs. Cependant, avant même que les idées de Canham n'aient pu recevoir un début de confirmation quantitative, de nombreuses interprétations alternatives ont vu le jour. Pour certains, une telle photoluminescence ne doit pas être attribuée au silicium, mais à des espèces chimiques superficielles. Ainsi des polysilanes [55] ou des siloxènes [56] ont été proposés comme des candidats possibles. Enfin, des variantes sont apparues pour les mécanismes faisant intervenir le semi-conducteur (excitons [57], états de surface [58] ...). En l'état actuel, les interprétations les plus monolithiques sont sans doute à exclure: il n'y a pas de polysilanes sur le silicium oxydé RTO, et pas de siloxènes sur le silicium dans HF, mais les effets de confinement quantiques sont sans doute impuissants à expliquer à eux seuls l'ensemble des observations; ainsi l'influence des différents traitements sur la recombinaison non radiative (passivation [59]) est sans doute à considérer. Il est difficile de trouver des techniques donnant des réponses permettant de trancher clairement, et le problème est compliqué par la multiplicité des paramètres de l'espace de préparation et la variété des matériaux étudiés dans les différents laboratoires. Une plus forte participation à cet effort de la communauté des électrochimistes serait sans doute utile.

Les tentatives de réaliser des dispositifs électroluminescents avec le silicium poreux n'ont jusqu'ici abouti qu'à de mauvais rendements (typiquement $10^{-5}$ ). Le problème essentiel semble résider dans la forte résistivité du matériau et la difficulté de maîtriser le lieu où se fait l'injection de porteurs (il est difficile de contacter la couche poreuse autrement qu'à sa surface externe). En revanche, une électroluminescence intense a été observée par voie électrochimique. Si on oxyde anodiquement une couche de silicium poreux, formé sur substrat $p$, dans un électrolyte aqueux exempt de fluorures, tel que $1 \mathrm{M} \mathrm{HCl}$ ou $1 \mathrm{M} \mathrm{KNO}_{3}$, on observe une électroluminescence intense pendant la phase d'oxydation [60]. Après passage d'une certaine charge, et bien que la totalité de la couche ne soit pas oxydée, la connexion entre le substrat et l'électrolyte se trouve rompue, et on ne peut plus faire passer de courant à des potentiels modérés; on perd donc l'électroluminescence, bien que le matériau obtenu à ce stade reste photoluminescent. On peut penser que l'électroluminescence résulte de l'injection d'électrons associée à un processus réactionnel en deux étapes, ici l'oxydation des liaisons $\mathrm{SiH}$ superficielles [schématiquement $\mathrm{SiH}+\mathrm{h}^{+} \rightarrow \mathrm{Si}^{+}+\mathrm{H}^{\circ}$ ads puis (avec $\mathrm{H}_{2} \mathrm{O}$ ) $\rightarrow \mathrm{SiOH}+\mathrm{H}_{2}+\mathrm{e}^{-}$] [43]. On a pu obtenir une électroluminescence plus durable sur silicium poreux formé sur substrat $n^{+}$, lors de la réduction cathodique de persulfate, système connu pour injecter facilement des trous $\left(\mathrm{S}_{2} \mathrm{O}_{8}{ }^{2-}+\mathrm{e}^{-} \rightarrow \mathrm{SO}_{4}{ }^{2-}+\mathrm{SO}_{4}{ }^{-\cdot} \rightarrow 2 \mathrm{SO}_{4}{ }^{2-}+\mathrm{h}^{+}\right)$[61,62]. Les bonnes performances de ces systèmes électrochimiques donnent l'espoir que des dispositifs solides pourront être réalisés à brève échéance.

\section{Electrochimie du silicium en milieu fluorure dilué: région de polissage.}

Le plateau de polissage observé pour le silicium en milieu fluorure a lui-même une forme fort complexe. On observe en fait deux plateaux; le premier, très étroit, est séparé du second par un maximum de courant assez large. La corrosion localisée apparaît entre 4 et $10 \mathrm{~V}$, suivant le type d'électrolyte, mais la forme qualitative du voltammogramme reste la même dans une large gamme de $\mathrm{pH}(0-8)$ et de concentration en fluorure $\left(10^{-2} \mathrm{M}-10 \mathrm{M}\right)[63]$. Une étude sur électrode tournante a montré que le processus faradique est sous contrôle mixte, et les courants cinétique et de diffusion ont pu être séparés [64]. Le courant de diffusion apparaît essentiellement gouverné par l'apport d'espèces fluorées $\left(\mathrm{F}^{-}, \mathrm{HF}_{1} \mathrm{HF}_{2}^{-}\right)$à l'électrode. Le courant cinétique présente une dépendance plus marquée en fonction de la concentration totale en fluorure $c_{F}\left(J_{K} \sim c_{F}^{\alpha}\right.$ avec 
$\alpha \approx 2$ dans une assez large gamme) et un maximum vers $\mathrm{pH} 3$, suggérant que l'espèce $\mathrm{HF}_{2}$ - joue un rôle important (mais non exclusif) dans le mécanisme.

L'étude de l'interface par spectroscopie infrarouge in-situ confirme que la surface est couverte de liaisons SiH jusqu'au premier pic de courant [65]. Sur le premier plateau, des pics caractéristiques de groupes $\mathrm{SiOH}$ apparaissent, mais une fraction des $\mathrm{SiH}$ subsistent, sous la forme d'un spectre élargi, suggérant la présence d'une couche superficielle désordonnée constituée d'un matériau du type $\mathrm{SiH}_{x}(\mathrm{OH})_{y}$. Les signatures d'un véritable oxyde n'apparaissent qu'audelà du second maximum de courant. Cet oxyde reste toutefois riche en défauts, comme l'atteste la présence d'un pic supplémentaire vers $1450 \mathrm{~cm}^{-1}$. Les mesures infrarouge indiquent d'autre part que des trous libres s'accumulent près de la

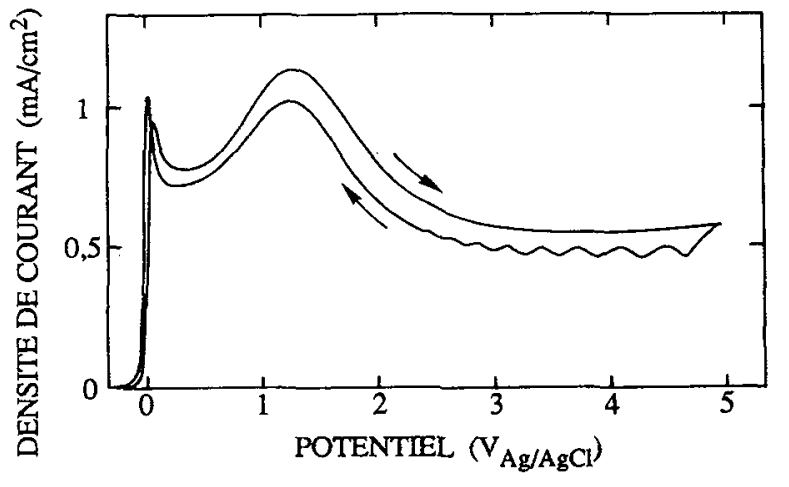

Fig.8.- Voltammogramme typique de $p$-Si en milieu fluorure (ici $\left.0.95 \mathrm{M} \mathrm{NH}_{4} \mathrm{Cl}+0.025 \mathrm{M} \mathrm{HF}+0.025 \mathrm{M} \mathrm{NH}_{4} \mathrm{~F}\right)$. Vitesse $20 \mathrm{mV} / \mathrm{s}$. Electrode tournante 300 tours/minute. surface essentiellement dans la région du second plateau. Une étude de transitoires de capacité a montré que dans cette région l'oxyde se charge positivement, ce qui accrédite l'idée d'un mécanisme de croissance par transport vers l'extérieur de charges positives, charges qui pourraient être associées à des lacunes d'oxygène [66].

Un aspect particulièrement surprenant de ce système électrochimique est le comportement électrique de l'interface dans la région du deuxième plateau. Le voltammogramme présente de petites oscillations de courant sur le trajet de retour du balayage. En fait, une étude montre qu'il ne s'agit pas là d'un comportement de l'interface à proprement parler oscillant, mais plutôt résonnant: en présence d'un potentiel constant, le système atteint un régime stationnaire, caractérisé par un courant constant. Toutefois, en présence d'une petite perturbation de potentiel, le courant présente un comportement transitoire oscillant, qui $s$ 'amortit lorsque la perturbation n'est plus appliquée [67]. La fréquence caractéristique de l'oscillation amortie $f_{0}$ varie avec la composition de l'êlectrolyte, en restant proportionnelle au courant stationnaire (typiquement $\mathrm{f}_{0} \sim 10^{-3}$ à $10^{2} \mathrm{~Hz}$ ). L'admittance du système présente un comportement résonnant pour la fréquence $\mathrm{f}_{0}$, mais aussi de façon inattendue pour les fréquences multiples $2 \mathrm{f}_{0}, 3 \mathrm{f}_{0}, \ldots$, bien que l'étude soit réalisée dans le régime de la réponse linéaire [68].

(b)

(a)

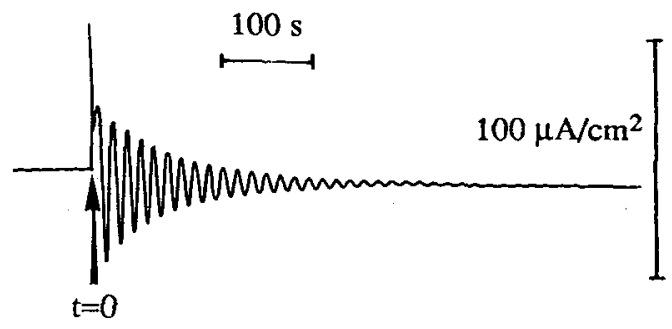

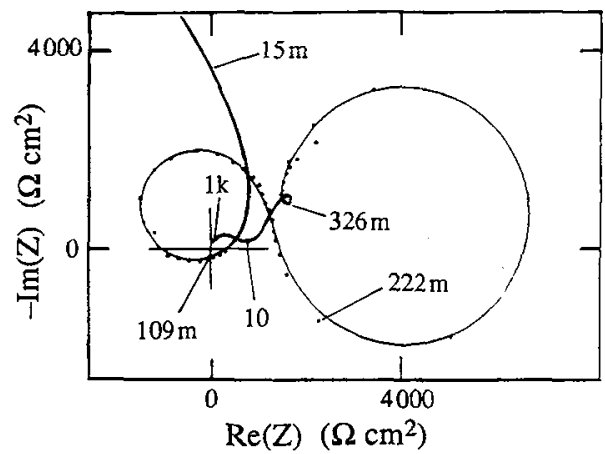

Fig.9.- Même système que Fig.8. (a) Oscillation amortie de courant obtenue en réponse à un petit saut de potentiel $+3,0 \rightarrow$ $+3,1 \mathrm{~V}$. La flèche indique l'instant du saut; (b) diagramme d'impédance pour $\mathrm{V}=+3,0 \mathrm{~V}$ (fréquences en $\mathrm{Hz}$ sur le diagramme). 
On a pu montrer que ce comportement de l'interface, semblable à celui d'une corde vibrante, s'interprète naturellement dans le cadre d'un modèle où le système est auto-oscillant à l'échelle microscopique, les différentes parties de la surface étant non corrélées: en régime stationnaire, l'absence de cohérence entre les différents "domaines" microscopiques fait que seul un courant moyen, constant, est observé. Une petite perturbation extérieure peut synchroniser partiellement les domaines, faisant apparaître une oscillation macroscopique; une fois la perturbation interrompue, les domaines reviennent progressivement à l'état incohérent, et le courant constant est recouvré. Une modélisation mathématique de ce phénomène permet de rendre compte de façon semi-quantitative des diagrammes d'impédance mesurés $[68,69]$. Des mesures de bruit électrochimique indiquent une taille caractéristique de $\sim 1000 \AA$ pour les domaines [70]. Par ailleurs, en présence d'une résistance série, ce comportement résonnant peut se transformer en comportement oscillant, précisément pour la valeur de résistance attendue d'après le diagramme d'impédance: il ne s'agit là que d'une synchronisation "artificielle" par le circuit extérieur à l'interface (composant discret ajouté en série ou résistance de l'électrolyte) [71]. Enfin, la fréquence de résonance étant proportionnelle à la densité de courant, le comportement résonnant peut constituer un outil simple pour l'étude de l'uniforme accessibilité de systèmes d'électrodes [72].

L'origine du comportement oscillant à l'échelle microscopique reste à déterminer. Bien qu'une controverse subsiste à ce sujet $[66,67,73,74]$, nous pensons, en nous appuyant sur des mesures de coulométrie, d'impédance, de transitoires, et d'absorption infrarouge, que l'oscillation à l'échelle microscopique est associé à une variation notable $(-50 \AA)$ de l'épaisseur de l'oxyde interfacial. L'épaisseur de l'oxyde résulte d'une compétition entre les mécanismes de formation par oxydation anodique (à l'interface silicium/oxyde) et de dissolution chimique (à la surface externe). Nous pensons que l'oscillation peut tirer son origine des défauts créés lors de la formation de l'oxyde. Lorsque ces défauts arrivent à la surface externe, la vitesse de dissolution est affectée, d'où une variation d'epaisseur,

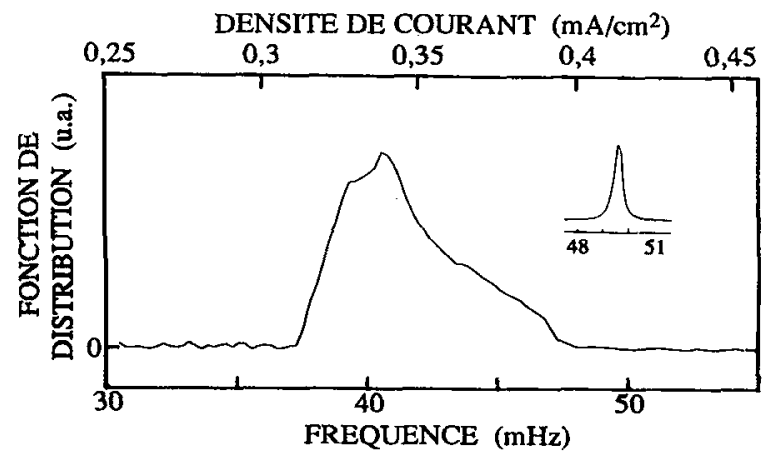

Fig.10.- Distribution de fréquence (échelle du bas) et distribution associée de courant (échelle du haut) à la surface d'une électrode dans une cellule à circulation, déterminé par analyse de Fourier de l'oscillation amortie de courant (d'après [72]). L'encart donne la forme obtenue sur une électrode uniformément accessible.

qui influe sur le courant anodique et affecte en retour la formation de nouveaux défauts. D'autres modèles ont également êté suggérés par d'autres auteurs [75]. Un travail plus quantitatif sur les différents modèles sera nécessaire avant qu'on puisse raisonnablement trancher.

\section{Conclusion.}

On pourrait penser que les propriétés du silicium, semi-conducteur élémentaire et matériau dominant de la microélectronique, sont parfaitement connues et comprises. Nous espérons avoir convaincu le lecteur qu'il n'en est rien. La chimie de surface et l'électrochimie du silicium, malgré quelques avenues bien tracées (tel le rôle de l'hydrogène à la surface, aspect si longtemps ignoré et désormais devenu incontournable), semblent de fait receler encore beaucoup de zones obscures. On peut penser que la curiosité des chercheurs, jointe à l'appât de riches applications potentielles, permettront dans les prochaines années de lever le voile sur quelques-unes de ces questions.

\section{Remerciements.}

Cette brève revue doit beaucoup à tous les auteurs dont les résultats ont été reproduits ici, en particulier $P$. Allongue et A. Halimaoui, qui ont aimablement fourni les clichés [Figs. 4 et 6], et à F. Ozanam, qui a effectué une relecture critique du manuscrit. 


\section{Références}

[1] voir par exemple a) Semiconductor Electrodes, Finklea H.O., éd. (Elsevier, Amsterdam, 1988); b) GERISCHER H., Electrochim. Acta 35 (1990) 1677.

[2] voir par exemple SERPONE N., BORGARELLO E., et PELIZZETTI E., in Photoelectrochemistry and Electrosynthesis on Semiconducting Materials, Ginley D.S., Nozik A., Armstrong N., Honda K., Fujishima A., Sakata T. et Kawai T., éd. (The Electrochemical Society Proc. 88-14, Pennington, 1988).

[3] LEWIS N.S., Ann. Rev. Mater. Sci. 14 (1984) 95.

[4] a) STIMMING U., Electrochim. Acta 31 (1986) 415; b) DI QUARTO F., AIMIUWU V.O., PIAZZA S. et SUNSERI C., Electrochim. Acta 36 (1991) 1817.

[5] voir par exemple LEHMANN V. et FOLL H., J. Electrochem. Soc. 135 (1988) 2831.

[6] NOTTEN P.H.L., VAN DEN MEERAKKER J.E.A.M., et KELLY J.J., in Etching of III-V Semiconductors: an Electrochemical Approach (Elsevier Advanced Technology, Oxford, 1991) ch.6.

[7] GALUS Z., in Standard Potentials in Aqueous Solution, Bard A.J., Parsons R. et Jordan J., éd. (Dekker, New York, 1985).

[8] voir par exemple LASER D. et BARD A.J., J. Phys. Chem. 80 (1976) 459.

[9] LEGG K.D., ELLIS A.B., BOLTS J.M. et WRIGHTON M.S., Proc. Natl. Acad. Sci. USA 74 (1977) 4116.

[10] OZANAM F. et CHAZALVIEL J.-N., J. Electroanal. Chem. 269 (1989) 251.

[11] GIBBONS J., COGAN G., GRONET C.M. et LEWIS N.S., Appl. Phys. Lett. 45 (1984) 1095.

[12] ABRAHAMS I.L., CASAGRANDE L.G., ROSENBLUM M.D., ROSENBLUTH M.L., SANTANGELO P.G., TUFTS B.F. et LEWIS N.S., New J. Chem. (Orsay) 11 (1987) 157.

[13] HARRICK N.J. et BECKMANN K.H., in Characterization of Solid Surfaces, Kane P.F. et Larrabee G.R., éd. (Plenum, New York, 1974) ch. 10, p. 243.

[14] UBARA H., IMARA T. et HIRAKI A., Solid State Comm. 50 (1984) 673.

[15] TARDELla A. et CHAZALVIEL J.-N., Appl. Phys. Lett. 47 (1985) 334.

[16] YABLONOVITCH E., ALLARA D.L., CHANG C.C., GMITTER T. et BRIGHT T.B., Phys. Rev. Lett. 57 (1986) 249.

[17] BURROWS V.A., CHABAL Y.J., HIGASHI G.S., RAGHAVACHARI K. et CHRISTMAN S.B., Appl. Phys. Lett. 53 (1988) 998.

[18] HIGASHI G.S., CHABAL Y.J., TRUCKS G.W. et RAGHAVACHARI K., Appl. Phys. Lett. 56 (1990) 656.

[19] HIGASHI G.S., BECKER R.S., CHABAL Y.J. et BECKER A.J., Appl. Phys. Lett. 58 (1991) 1656.

[20] DUMAS P. et CHABAL Y.J., Chem. Phys. Lett. 181 (1991) 537.

[21] GUYOT-SIONNEST P., Phys. Rev. Lett. 67 (1991) 2323.

[22] MORIN R., JAKOB P., LEVINOS N.J., CHABAL Y.J. et HARRIS A.L., J. Chem. Phys. 96 (1992) 6203.

[23] ILER R.K., The Chemistry of Silica (Wiley, New York, 1979).

[24] YONEYAMA H., MURAO Y. et TAMURA H., J. Electroanal. Chem. 108 (1980) 87.

[25] CHAZALVIEL J.-N., J. Electroanal. Chem. 233 (1987) 37.

[26] WRIGHTON M.S., AUSTIN R.G., BOCARSLY A.B., BOLTS J.M., HAAS O., LEGG K.D., NADJO L. et PALAZZOTTO M.C., J. Am. Chem. Soc. 100 (1978) 1602.

[27] SEIDEL H., CSEPREGI L., HEUBERGER A. et BAUMGARTEL H., J. Electrochem. Soc. 137 (1990) 3612.

[28] PALIK E.D., BERMUDEZ V.M. et GLEMBOCKI O.J., J. Electrochem. Soc. 132 (1985) 871.

[29] ALlONGUE P., BRUNE H. et GERISCHER H., Surf. Sci. 275 (1992) 414.

[30] ALLONGUE P., KIELING V. et GERISCHER H., J. Electrochem. Soc. 140 (1993, à paraître).

[31] SCHMIDT P.F. et MICHEL W., J. Electrochem. Soc. 104 (1957) 230.

[32] MADOU M.J., MORRISON S.R. et BONDARENKO V.P., J. Electrochem. Soc. 135 (1988) 229.

[33] GASPARD F., HALIMAOUI A. et SARRABAYROUSE G., Revue Phys. Appl. 22 (1987) 65.

[34] MEMMING R. et SCHWANDT G., Surf. Sci. 4 (1966) 109.

[35] ZHANG X.G., COLLINS S.D. et SMITH R.L., J. Electrochem. Soc. 136 (1989) 1561.

[36] MATSUMURA M. et MORRISSON S.R., J. Electroanal. Chem. 147 (1982) 157.

[37] BLACKWOOD D.J., BORAZIO A., GREEF R., PETER L.M. et STUMPER J., Electrochim. Acta 37 (1992) 889.

[38] LEWERENZ H.J., STUMPER J. et PETER L.M., Phys. Rev. Lett. 61 (1988) 1989.

[39] GERISCHER H. et LUBKE M., Ber. Bunsenges. Phys. Chem. 92 (1988) 573.

[40] GERISCHER H. et LUBKE M., J. Electrochem. Soc. 135 (1988) 2782. 
[41] EDDOWES M.J., J. Electroanal. Chem. 280 (1990) 297.

[42] VENKATESWARA RAO A., OZANAM F. et CHAZALVIEL J.-N., J. Electrochem. Soc, 138 (1991) 153.

[43] CHAZALVIEL J.-N. et OZANAM F,, in Microcrystalline Semiconductors - Material Science and Device, Aoyagi Y., Canham L.T., Fauchet P., Shimizu I. et Tsai C.C., éd., MRS Symp. Proc. 283 (1993) 359.

[44] LEHMANN V. et GOSELE U., Appl. Phys. Lett. 58 (1991) 856.

[45] GERISCHER H., ALLONGUE P. et KIELING V., Ber. Bunsenges. Phys. Chem. (1993, à paraître).

[46] UHLIR A., Bell. Syst. Tech. J. 35 (1956) 333.

[47] TURNER D.R., J. Electrochem. Soc. 105 (1958) 402.

[48] BOMCHIL G., HALIMAOUI A. et HERINO R., Appl. Surf. Sci. $41 / 42$ (1989) 604.

[49] a) SMITH R.L. et COLLINS S.D., J. Appl. Phys. 71 (1992) R1; b) BOMCHIL G., HALIMAOUI A., SAGNES I., BADOZ P.A., BERBEZIER I., PERRET P., LAMBERT B., VINCENT G., GARCHERY L. et REGOLINI J.L., Appl. Surf. Sci. $65 / 66$ (1993) 394.

[50] UNAGAMI T., J. Electrochem. Soc. 127 (1980) 476.

[51] BEALE M.I.J., CHEW N.G., UREN M.J., CULLIS A.G. et BENJAMIN J.D., Appl. Phys. Lett. 46 (1985) 86.

[52] CANHAM L.T., Appl. Phys. Lett. 57 (1990) 1046.

[53] BSIESY A., VIAL J.-C., GASPARD F., HERINO R., LIGEON M., MULLER F., ROMESTAIN R., WASIELA A., HALIMAOUI A. et BOMCHLL G., Surf. Sci. 254 (1991) 195.

[54] PETROVA-KOCH V., MUSCHIK T., KUX A., MEYER B.K., KOCH F. et LEHMANN V., Appl. Phys. Lett. 61 (1992) 943.

[55] PROKES S.M., GLEMBOCKI O.J., BERMUDEZ V.M., KAPLAN R., FRIEDERSDORF L.E. et SEARSON P.C., Phys. Rev. B 45 (1992) 13788.

[56] BRANDT M.S., FUCHS H.D., STUTZMANN M., WEBER J. et CARDONA M., Solid State Comm. 81 (1992) 307.

[57] CALCOTT P.D.J., NASH K.J., CANHAM L.T., KANE M.J. et BRUMHEAD D., Solid State Comm. (1993, à paraître).

[58] PETROVA-KOCH V., MUSCHIK T., GAVRILENKO V. et KOCH F., in Microcrystalline Semiconductors - Material Science and Device, Aoyagi Y., Canham L.T., Fauchet P., Shimizu I. et Tsai C.C., Ed., MRS Symp. Proc. 283 (1993, à paraître).

[59] VIAL J.-C., BILLAT S., BSIESY A., FISHMAN G., GASPARD F., HERINO R., LIGEON M., MADEORE F, MIHALCESCU I., MULLER F. et ROMESTAIN R., Physica B (1993, à paraître).

[60] HALIMAOUI A., OULES C., BOMCHIL G., BSIESY A., GASPARD F., HERINO R., LIGEON M. et MULLER F., Appl. Phys. Lett. 59 (1991) 304.

[61] BRESSERS P.M.M.C., KNAPPEN J.W.J., MEULENKAMP E.A. et KELLY J.J., Appl. Phys. Lett. 61 (1992) 108.

[62] CANHAM L.T., LEONG W.Y., BEALE M.I.J., COX T.I. et TAYLOR L., Appl. Phys. Lett. 61 (1992) 2563.

[63] CHAZALVIEL J.-N., ETMAN M. et OZANAM F., J. Electroanal. Chem. 297 (1991) 533.

[64] ETMAN M., NEUMANN-SPALLART M., CHAZALVIEL J.-N. et OZANAM F., J. Electroanal. Chem. 301 (1991) 259.

[65] CHAZALVIEL J.-N., DUBIN V.M., MANDAL K.C. et OZANAM F., Appl. Optics (1993, à paraître).

[66] CHAZALVIEL J.-N., Electrochim. Acta 37 (1992) 865.

[67] OZANAM F., CHAZALVIEL J.-N., RADI A. et ETMAN M., Ber. Bunsenges. Phys. Chem. 95 (1991) 98.

[68] OZANAM F., CHAZALVIEL J.-N., RADI A. et ETMAN M., J. Electrochem. Soc. 139 (1992) 2491.

[69] CHAZALVIEL J.-N. et OZANAM F., J. Electrochem. Soc. 139 (1992) 2501.

[70] OZANAM F., BLANCHARD N. et CHAZALVIEL J.-N., Electrochim. Acta (1993, à paraître).

[71] CHAZALVIEL J.-N., OZANAM F., ETMAN M., PAOLUCCI F., PETER L.M. et STUMPER J., J.Electroanal. Chem. 327 (1992) 343.

[72] CHAZALVIEL J.-N. et OZANAM F., Ber. Bunsenges. Phys. Chem. 96 (1992) 1809.

[73] STUMPER J. et PETER L.M., J. Electroanal. Chem. 309 (1991) 445.

[74] LEWERENZ H.J. et SCHLICHTHORL G., J. Electroanal. Chem. 327 (1992) 85.

[75] FOLL H., Appl. Phys. A 53 (1991) 8. 